Musées, Patrimoine et Culture scientifiques et techniques

$157 \mid 2015$

janvier-février 2015

\title{
Des conservations départementales aux coordinations départementales des musées
}

\author{
Cécile Massot
}

\section{OpenEdition \\ Journals}

Édition électronique

URL : http://journals.openedition.org/ocim/1469

DOI : $10.4000 /$ ocim. 1469

ISSN : 2108-646X

Éditeur

OCIM

Édition imprimée

Date de publication : 1 janvier 2015

Pagination : 5-11

ISSN : 0994-1908

Référence électronique

Cécile Massot, « Des conservations départementales aux coordinations départementales des musées », La Lettre de I'OCIM [En ligne], 157 | 2015, mis en ligne le 01 janvier 2016, consulté le 01 mai 2019 URL : http://journals.openedition.org/ocim/1469 ; DOI : 10.4000/ocim.1469

Ce document a été généré automatiquement le 1 mai 2019.

Tous droits réservés 


\title{
Des conservations départementales aux coordinations départementales des musées
}

\author{
Cécile Massot
}

Les départements dotés de coordinations départementales des musées en 2013 sont représentés en vert.

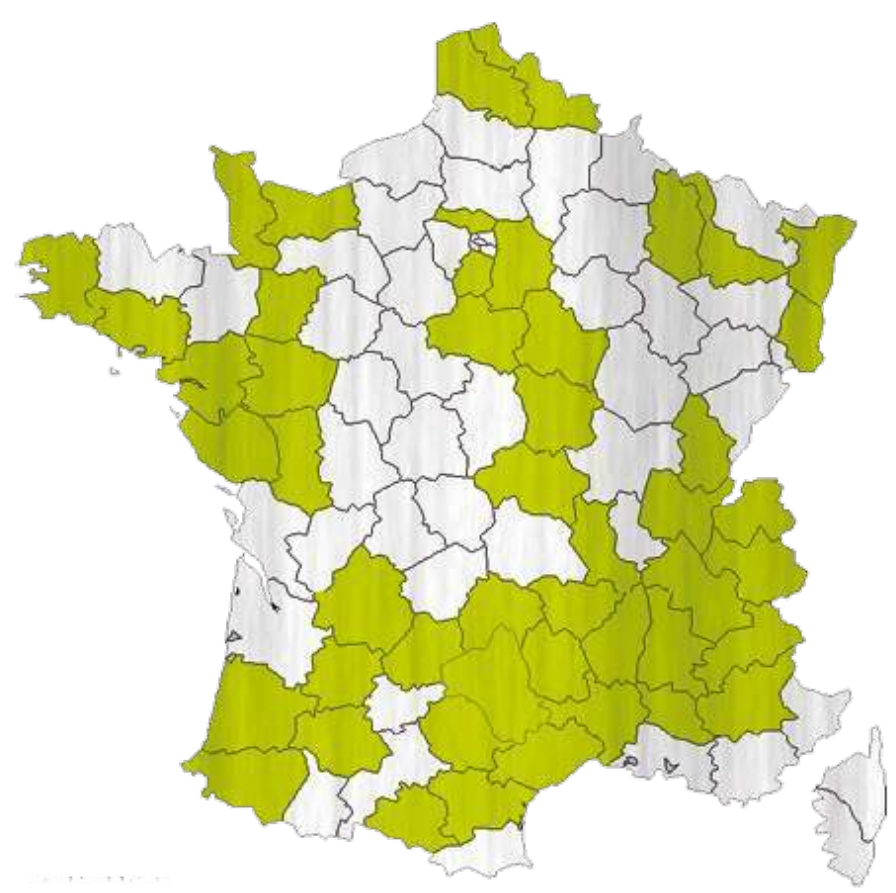


1 Le nombre des petites structures muséales, propriétés de communes ou d'associations, n'a cessé de grandir au cours de la deuxième moitié du XX $\mathrm{X}^{\mathrm{e}}$ siècle. Les propriétaires de ces musées n'ont souvent ni les moyens, ni les compétences nécessaires pour leur développement ou simplement leur survie. Depuis le milieu des années 1950, certains se tournent vers l'administration de leur Département pour demander une assistance financière et méthodologique. Une petite moitié des Départements français a alors fait le choix de créer une conservation départementale des musées.

2 Les premières conservations départementales des musées ont vu le jour dans les années 1950. Le premier service de ce genre, créé dans le contexte d'après-guerre en 1955, est celui de l'Inspection départementale des musées contrôlés du Calvados (service d'État, qui deviendra départemental en 1982). En 1956, c'est la Meuse qui se dote d'une conservation départementale des musées, puis le Jura au début des années 1960. Quatre conservations (Vendée, Haute-Marne, Landes, Hérault) vont voir le jour au cours de la décennie 1970. Mais c'est principalement dans les années 1980 et 1990, à la suite des lois de décentralisation, que les conservations départementales des musées ont été créées : huit services existaient en 1979, vingt-trois en 1989, quarante-cinq en 1999. Le développement du nombre de conservations départementales des musées s'est effectué en continu, il y en a aujourd'hui une cinquantaine.

3 Il est important d'avoir à l'esprit que les conservations départementales des musées ne sont pas des institutions définies publiquement et légalement. Elles sont apparues au fil du temps et ont connu une évolution hétérogène. Si le ministère de la Culture reconnaît leur existence, il ne fait que constater l'existant sans légiférer, ni donner de consignes de travail ou de direction d'évolution. De ce fait, les conservations départementales des musées n'ont pas de définition officielle. Ceci entraîne inévitablement une très grande diversité liée aux différences de besoins, de fonctionnement, de politique culturelle départementale... Cette variété se retrouve, par exemple, dans les noms que se sont donnés les services départementaux : conservation départementale, service/conservation des musées, coordination des musées municipaux et associatifs, réseau des musées, développement des musées, service de l'action culturelle, service/mission patrimoine, conservation du patrimoine...

4 Dans le cadre de cette étude, il était indispensable de définir un cadre clair permettant d'identifier tel service comme relevant d'une conservation départementale des musées et non tel autre. En s'appuyant sur les études préexistantes, nous avons délimité le terme de conservation départementale des musées à un service d'un Département (ou une personne dépendant d'un service départemental) ayant pour missions et de manière pérenne une ou plusieurs des tâches suivantes :

5 - le conseil et l'expertise méthodologique ou scientifique, en ce qui concerne les musées, écomusées, espaces muséographiques auprès des communes et associations du département ;

6 - la responsabilité scientifique, technique et/ou administrative de musées non départementaux dépourvus de conservateur affecté à l'établissement ;

7 - l'accompagnement des projets de (re)structuration, de programmation, de chantier des collections... réalisés par les communes, les associations ou les privés pour leurs musées (ou leurs collections) ;

8 - la mise en réseau de plusieurs institutions muséales présentes sur le territoire départemental. 
9 En se limitant à cette définition, quarante-sept coordinations départementales des musées ont été recensées.

\section{Création, évolution et fonctionnement des conservations départementales des musées}

\section{Le contexte de création}

10 Les principales raisons de la création des conservations départementales des musées correspondent à la demande des communes de déléguer des missions trop lourdes pour elles. Les notions de soutien et de solidarité des Départements envers les structures plus petites de leur territoire sont très importantes. En effet, la légitimité des coordinations départementales des musées repose sur l'article 27 de la loi n $82-213$ relative aux libertés des Communes, des Départements et des Régions : "Le Département apporte aux Communes qui le demandent son soutien à l'exercice de leur compétence".

Déclencheurs pour la création des coordinations départementales des musées

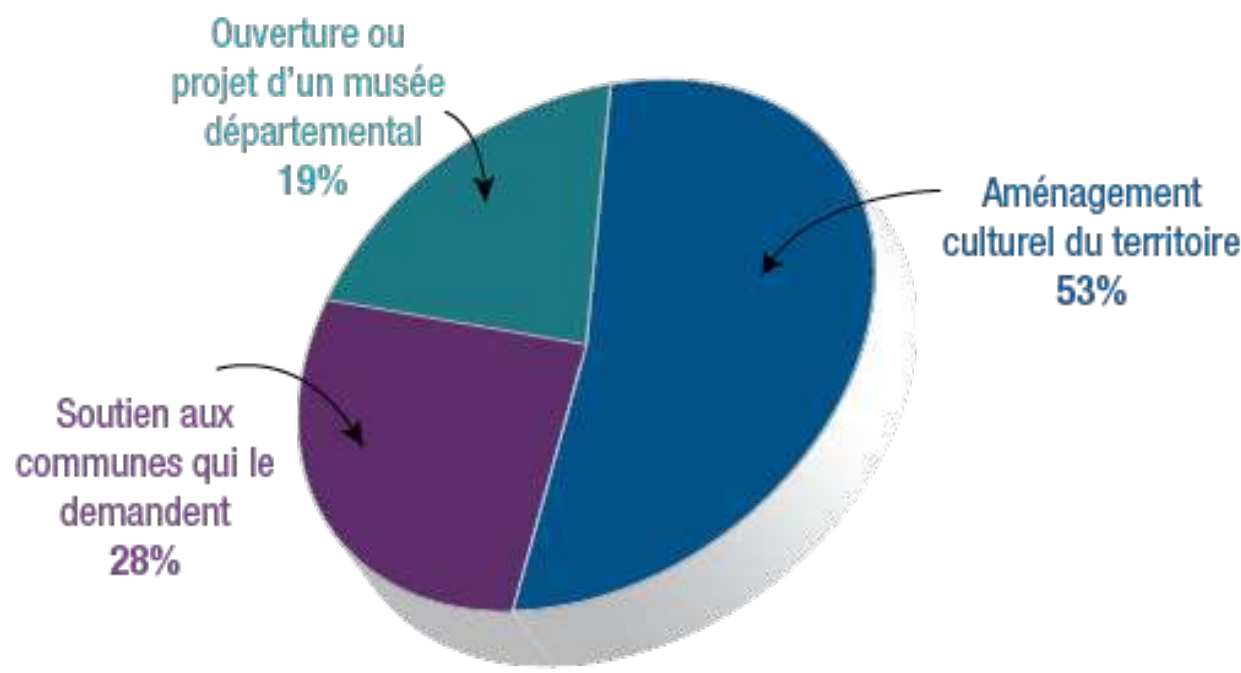

\section{(c) Cécile Massot}

Quelques chiffres issus de notre étude permettent d'illustrer ce propos :

12 - près d'un tiers des services a été créé pour apporter "un soutien aux communes qui le demandent" ;

$13-20 \%$ des coordinations départementales des musées dépendent d'une Direction portant le terme de Solidarité ou Citoyenneté ;

$14-40 \%$ des coordinations déclarent avoir pour mission la gestion directe d'au moins un musée non départemental ou la mise à disposition de personnel départemental pour un musée du territoire. 
Compétences des directions auxquelles sont rattachées les coordinations départementales des musées

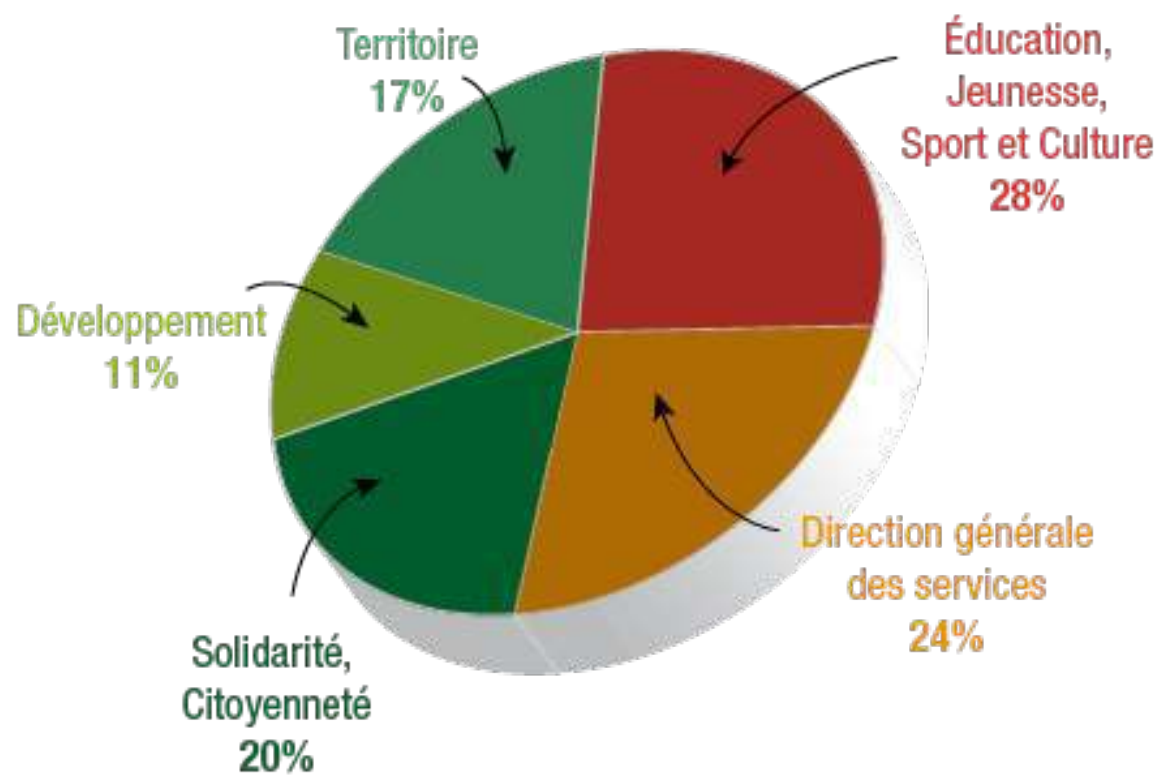

(c) Cécile Massot 


\section{Une mutation au fil du temps}

19 L'évolution des conservations départementales des musées vers cette direction est très intéressante et lisible dans les différentes études ${ }^{1}$. La première étude qui date de 1992 ne fait que très peu référence à l'aménagement du territoire, elle s'attache surtout à recenser les Départements apportant, ponctuellement ou durablement, une expertise scientifique aux petits musées². La grande étude réalisée en 1999 montre que "les conservations départementales (...) participent activement aux stratégies d'aménagement, contribuent au renforcement de l'attractivité du territoire et au développement qualitatif et quantitatif du tourisme culturel"3.

En 2005, un état des lieux sur les conservations départementales identifie vingt-deux services ayant un réseau de musées et plus d'une dizaine qui "mettent en place une stratégie de développement patrimonial"4.

21 Enfin, en 2012, lors des journées d'étude et de formation organisées par l'AGCCPF sur les réseaux, les conservateurs départementaux de Midi-Pyrénées ont choisi de définir une conservation départementale comme répondant "au schéma: des collections, un territoire, un réseau"5.

Campagne de prise de vue des collections antiques du musée du Pays de Château-Gontier, à l'aide du studio photographique nomade de la conservation départementale de Mayenne.

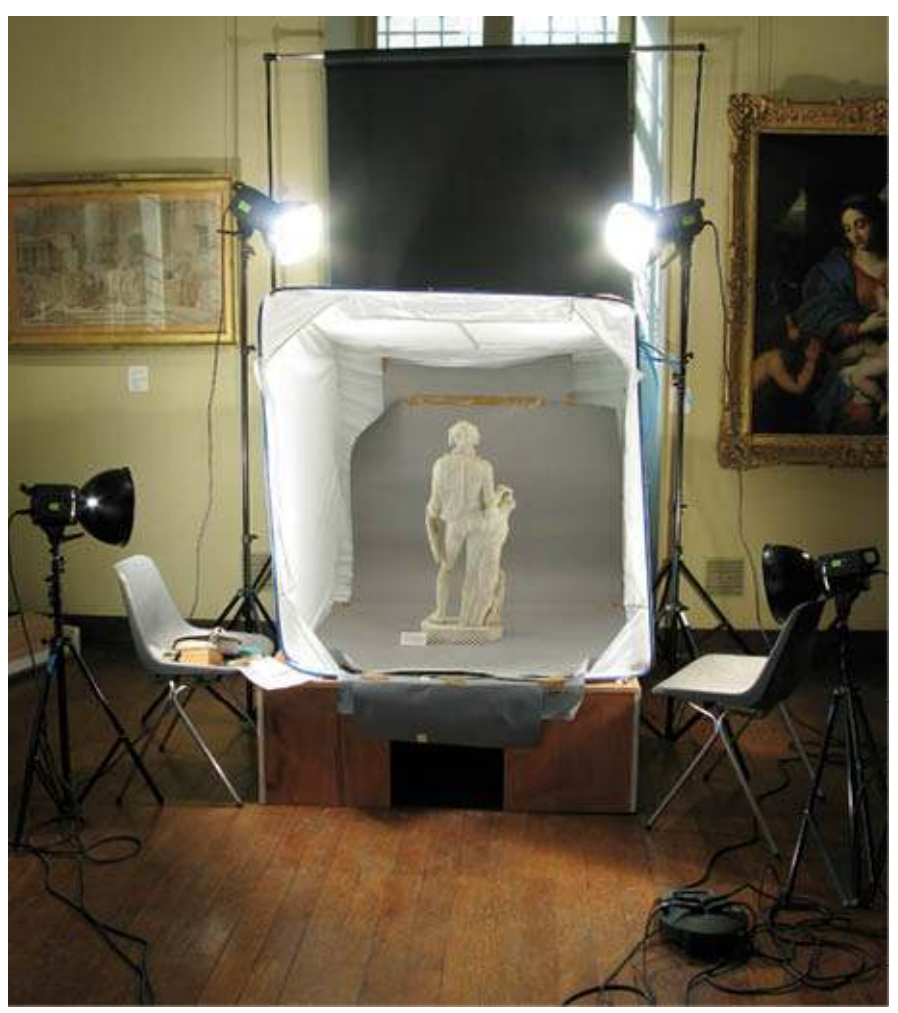

(c) Jean Mathiaut

Les résultats de l'enquête réalisée en 2013 montrent une réelle inflexion de la politique des conservations départementales des musées vers une réflexion globale sur les musées du territoire et l'aide ou le soutien à leur apporter. Les conservations placent désormais au centre de leurs préoccupations la dynamique muséale du territoire, les interactions 
entre les musées et leurs publics, la méthodologie de projet et de mutualisation. En vingt ans, les conservations départementales sont ainsi majoritairement passées d'un travail individualisé avec chaque musée demandeur à une politique muséale globale travaillant avec le territoire dans son ensemble. Ce qui n'exclut pas le travail au cas par cas avec les musées mais ce travail se place au sein d'un projet à plus grande échelle. Les conservations départementales sont devenues un outil de développement local.

Il est d'ailleurs remarquable et significatif que près de $30 \%$ des Départements aient aujourd'hui rattaché leur coordination départementale des musées à une direction portant un nom se référant au "développement", à "l'aménagement" ou au "territoire". La mutation des conservations départementales des musées amène à la conclusion qu'elles ne sont plus des conservations mais de véritables coordinations. Le terme conservation définit le maintien d'une activité, l'action de faire que quelque chose reste en bon état, continue d'avoir la même qualité. Le mot coordination se réfère lui à la combinaison d'actions, la constitution d'un ensemble cohérent, le fait d'ordonner ou de relier des éléments. Le nom de coordination départementale des musées semble donc beaucoup plus approprié à la situation actuelle des services étudiés (missions, fonctionnements, actions).

Mission des coordinations départementales des musées

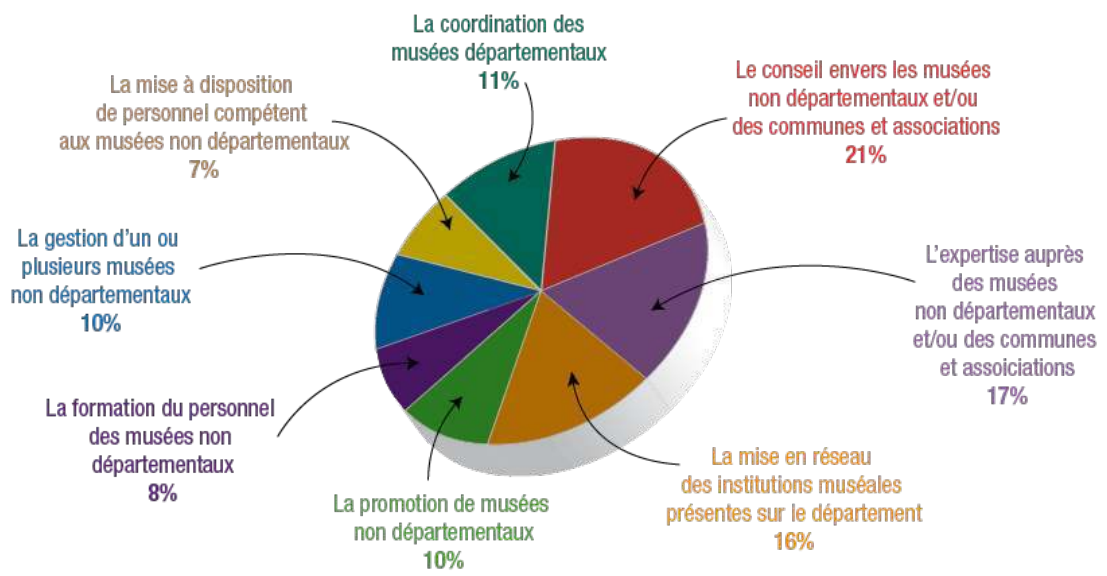

(c) Cécile Massot

\section{Les différentes missions} construction d'une méthodologie de projet. Les coordinateurs sont identifiés comme les experts muséaux du territoire, vers qui les structures demandeuses peuvent se tourner. Ce sont des personnes qualifiées dans la mise en valeur du patrimoine et dans la gestion de projets culturels. Elles sont aptes à conseiller et à accompagner les initiatives locales. Le conseil et l'expertise auprès des musées du territoire occupent environ $40 \%$ des temps de travail des coordinations départementales ; 
27 - les coordinations départementales des musées participent également au développement territorial par la formation et la professionnalisation des équipes. C'est une mission qui apparaît assez faiblement dans les résultats chiffrés de l'enquête, sans doute en raison d'une mauvaise terminologie dans l'étude, mais qui est en fait au cœur des problématiques de ces services. Les coordinations départementales des musées tendent de plus en plus à travailler vers la professionnalisation afin de ne plus créer de situation de dépendance entre les petites structures muséales et les Départements. Une conservatrice départementale a par exemple clairement exprimé l'objectif de sa fonction : que les musées puissent voler de leurs propres ailes et qu'ils n'aient plus besoin d'elle ;

28 - enfin, le développement passe parfois par un soutien à la communication des musées ou des réseaux de musées. Cependant, cette mission est assez peu confiée aux coordinations départementales des musées, souvent par manque de budget.

\section{Développement et aménagement du territoire}

Galerie d'exposition du musée des Arts et Métiers traditionnels de Salles-la-Source, géré par le service des musées départementaux d'Aveyron

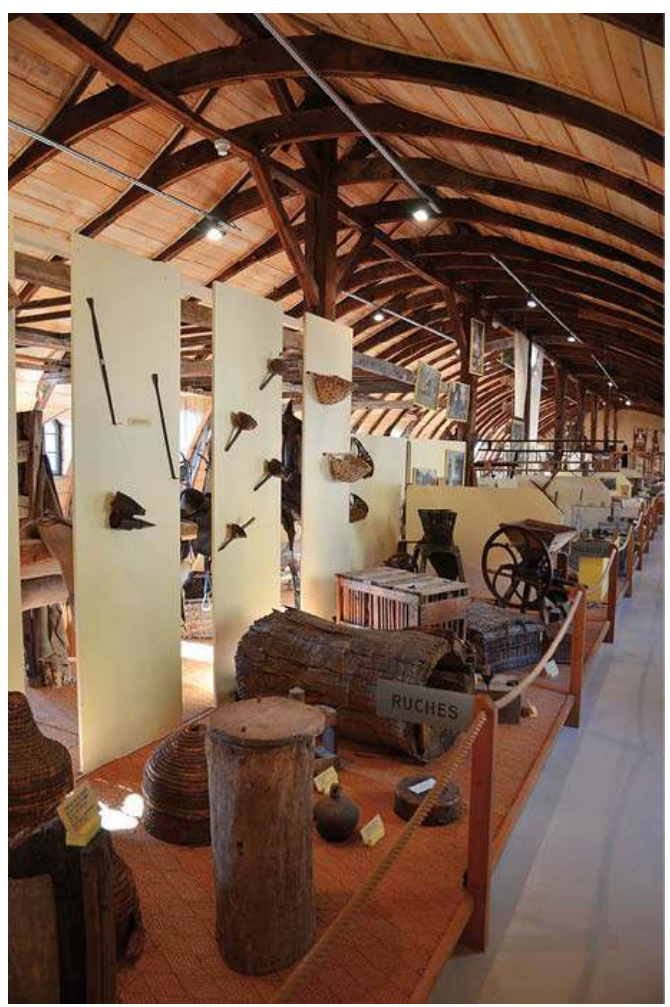

(C) Photothèque du Conseil général de l'Aveyron $\mathrm{du}$ territoire, c'est-à-dire l'organisation et l'équilibre de l'ensemble des projets muséographiques. Les missions des conservateurs départementaux "relèvent de la coordination, de l'harmonisation et de la structuration d'une offre muséale et patrimoniale qui doit s'inscrire dans une politique culturelle départementale"6.

Alors que plus de la moitié des coordinations départementales affirment participer à l'aménagement culturel de leur territoire, l'enquête a mis en lumière le fait que 
seulement cinq ont mis en place un schéma muséographique départemental et onze un projet de service clairement établi. Cette absence de direction pour de nombreuses coordinations départementales, et même parfois l'absence de réflexion réelle au niveau des élus du Département sur le fonctionnement de ces services, entrave la mise en place d'un aménagement dynamique du territoire muséal, c'est-à-dire d'un territoire culturellement irrigué et réticulé. Il reste que, comme Fabrice Denise le constatait dans son étude en 1999, "les conservations départementales remplissent un vide. Elles sont les seuls organismes pouvant prendre en charge et améliorer sensiblement des musées locaux non contrôlés mais qui existent et peuvent posséder des collections de qualités. Ces services réalisent le maillage d'un territoire: ils constituent un filtre permettant de rationaliser une multitude de projets et de sélectionner selon des critères de pertinence et de complémentarité les sites structurants du département".

Les témoignages des conservateurs départementaux vont dans ce sens : "l'accompagnement ou non de certains projets de musées induit [inévitablement] une orientation et un contrôle du paysage muséal départemental" (Sarret, 2002); la coordination départementale des musées répond au besoin "d'harmonisation des Musées de France du département dans un souci de cohérence en évitant les doublons et la concurrence entre eux" (Ricot, 2013); le service a pour enjeux "la lisibilité et la mise en cohérence des politiques culturelles, ainsi que le développement et le rééquilibrage territorial" (Lecat, 2013).

\section{Les coordinations départementales : des réseaux territoriaux ?}

\section{Qu'est-ce qu'un réseau?}

Lorsqu'on parle d'aménagement territorial, la notion de réseau vient immédiatement à l'esprit, un territoire est aménagé grâce aux réseaux qu'on y déploie, le réseau est un principe d'aménagement du territoire. Les coordinations départementales sont-elles alors des réseaux ? Elles s'identifient et sont souvent qualifiées comme telles. La mise en réseau des institutions muséales présentes sur le département apparaît d'ailleurs en troisième position des missions confiées aux coordinations départementales des musées. Pourtant la confrontation des résultats de l'enquête à des ouvrages scientifiques et philosophiques sur la notion de réseau nous a beaucoup questionnée.

Pierre Musso (1997), qui a centré sa recherche sur la notion de réseau, identifie par exemple le réseau à un filet ; "la première représentation empirique d'un réseau est un ensemble de lignes et d'interconnexions, de chemins et de sommets"7.

L'application de cette définition aux coordinations des musées ferait que tous les musées, avec lesquels chaque service départemental travaille mais aussi la coordination seraient les points et que les relations qui existent entre chacun seraient les liens. Cependant dans l'organisation de travail des coordinations départementales avec les musées du territoire, les liens sont souvent propres à la collaboration entre le service du Conseil général et chaque musée. Aussi, la coordination départementale, sans être hiérarchiquement placée au-dessus, a développé une position d'expertise et de conseil, elle n'est donc pas sur le même niveau que tous les autres points du possible réseau. 
Réunion semestrielle de la coordination du Département du Nord dans le cadre du projet européen FLAMUSE au musée des Jeux traditionnels de Loon-Plage, janvier 2011

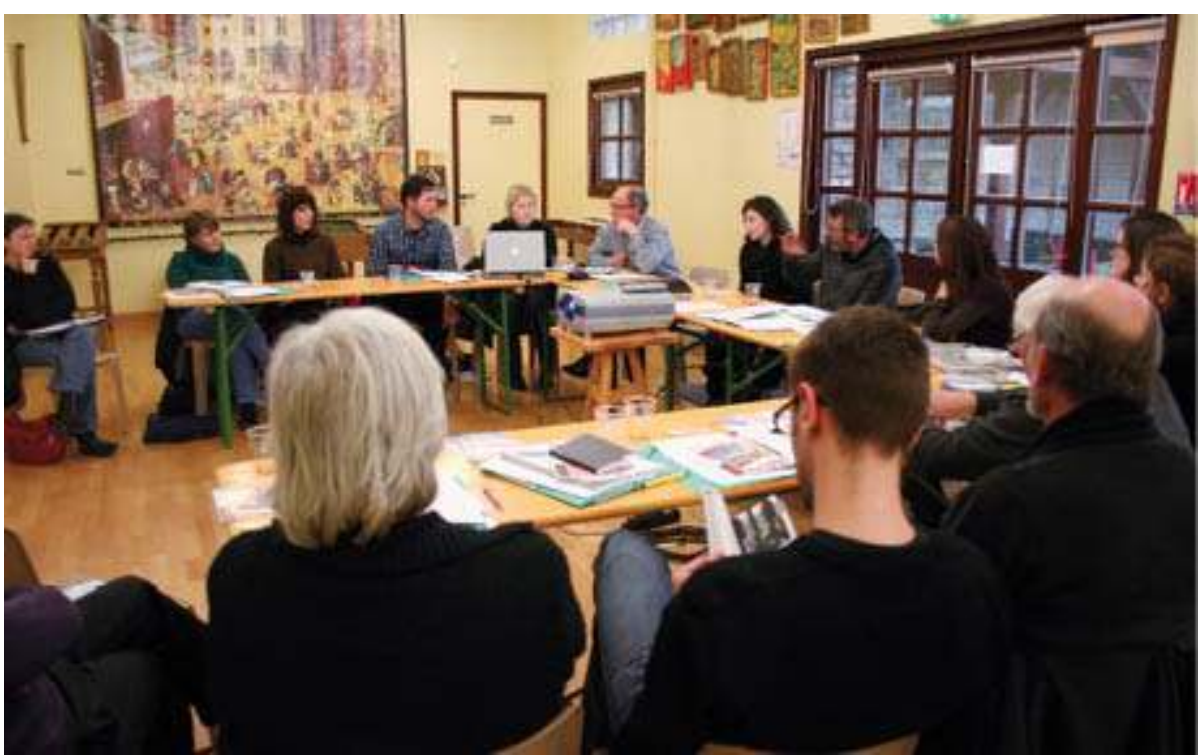

(c) Département du Nord/C. Fleury réseaux? fréquents. principaux :

Mais les réseaux de musées ont aussi été définis "comme un regroupement de différents établissements, articulés, la plupart du temps, autour d'une structure fédératrice"8.

Les coordinations départementales pourraient-elles alors être considérées comme des

Un réseau est un maillage, un filet, c'est une machine relationnelle. Le réseau est un outil de travail qui permet à ses membres d'échanger entre eux, de travailler en commun, de mutualiser leurs efforts. Pourtant, l'étude des fonctionnements des territoires avec une coordination départementale des musées montre que les relations entre les musées passent de manière générale par la coordination. Les cas où les musées travaillent en commun ou en mutualisation sans passer par le service départemental sont peu

\section{Les différents fonctionnements des coordinations départementales des musées}

Pour approfondir l'analyse, il faut étudier au plus près le fonctionnement de chaque coordination départementale et ses rapports avec et entre les musées avec lesquels elles travaillent. Dans le souci de clarifier notre propos, nous avons identifié quatre schémas

9 - un fonctionnement prescripteur: la coordination départementale des musées assure principalement des missions de conseils et d'expertise auprès des musées du territoire. Elle n'a pas de schéma muséographique ou d'axe de développement défini à suivre, elle répond uniquement aux sollicitations des acteurs culturels locaux. Dix-neuf coordinations départementales des musées de ce type ont été recensées ;

40 - un fonctionnement centralisateur: les coordinations départementales travaillent de manière autonome avec chaque musée mais suivent un projet de service, une politique 
culturelle départementale clairement établie. Chaque action s'inscrit donc dans une dynamique globale et selon des orientations prédéfinies. Une quinzaine de coordinations départementales des musées fonctionnent ainsi ;

41 - un fonctionnement animateur: la coordination des musées est ici l'initiatrice d'un réseau de musées, qui travaillent ensemble, principalement pour ce qui concerne la communication et des actions événementielles ponctuelles. Elle est l'animatrice de ce réseau, c'est grâce à elle qu'il existe et qu'il est vivant. La coordination est force de propositions et d'organisation, souvent aussi de financement. Dans la majorité des cas, le réseau ne survivrait pas à sa disparition. Seules huit coordinations départementales se sont révélées suivre ce schéma ;

42 - un fonctionnement souteneur : la coordination départementale des musées n'est pas membre d'un réseau de musées mais favorise et facilite la mise en réseau des musées du territoire. Le service départemental peut soutenir financièrement les réseaux, leur proposer un accompagnement scientifique ou méthodologique...

43 Nous avons dénombré cinq coordinations départementales des musées allant dans ce sens.

Répartition des coordinations départementales des musées selon les fonctionnements

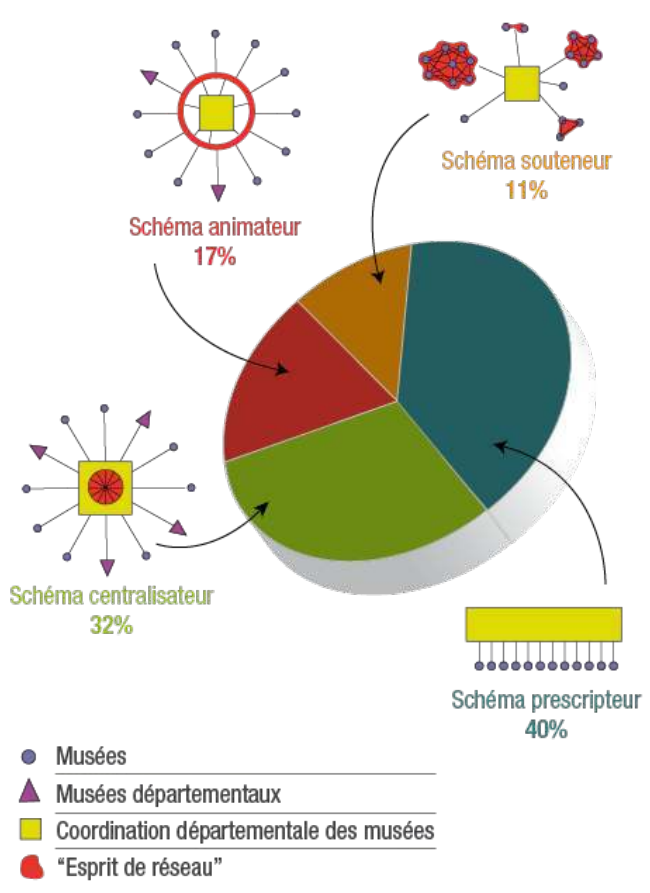

(c) Cécile Massot

Les quatre schémas correspondant aux quatre fonctionnements montrent distinctement que les coordinations départementales ne peuvent pas être définies systématiquement comme des réseaux: les zones rouges, "l'esprit de réseau", ne sont pas majoritaires. Moins d'un cinquième des coordinations départementales des musées est réellement au cœur d'un réseau auquel tous les musées du territoire participent. Un dixième contribue à leur développement mais n'en fait pas intégralement partie. Un tiers des coordinations 
départementales fonctionne avec des interconnexions qui se font uniquement en interne; l'étude et l'analyse des actions menées montrent que la coordination suit un schéma directeur, mais celui-ci n'est pas écrit : comme ce sont les mêmes personnes qui dirigent ou qui sont les responsables scientifiques des différents musées, elles appliquent simplement la même méthodologie et une politique muséale proche. Enfin, le schéma qui regroupe le plus de coordinations départementales de musées est celui où "l'esprit de réseau" est totalement absent du fonctionnement, les actions se font au cas par cas.

Une des missions des coordinations départementales : formation en conservation préventive au Fort Leveau de Feignies avec SBV Conservation consulting, décembre 2012

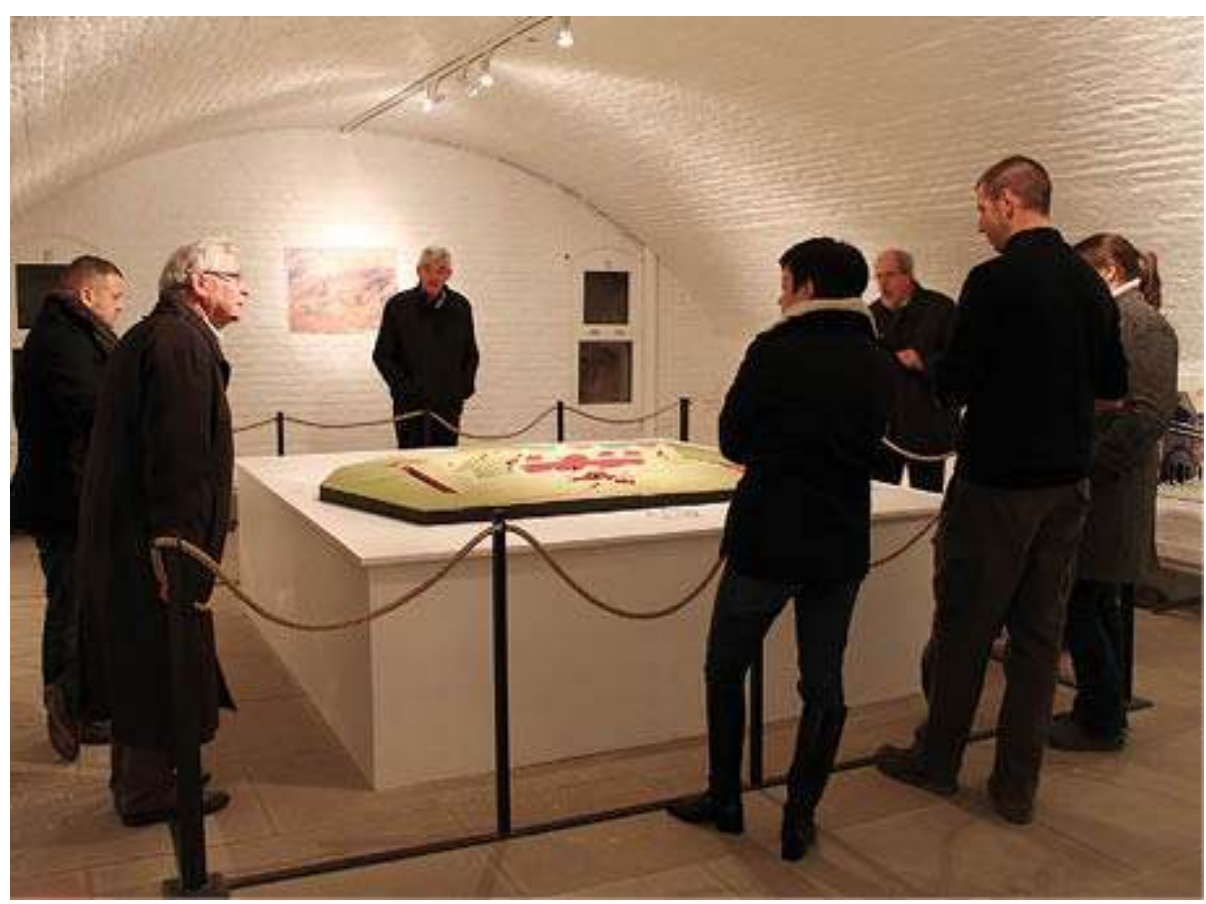

(c) Département du Nord/C. Fleury

Finalement, l'enquête montre que les coordinations départementales des musées sont des carrefours de rencontres, mais ne constituent pas pour autant systématiquement des réseaux de musées. Elles permettent d'organiser le territoire, de favoriser le développement d'actions culturelles, d'établir des relations entre le Département et les musées du territoire ; mais l'aménagement du territoire se fait parfois de manière uniaxiale (du Département vers les musées) et non dans une logique évidente de mise en relation des "musées associés" : ce terme, très souvent employé dans les études liées aux coordinations départementales des musées, illustre bien notre propos; les musées du territoire sont considérés comme des institutions associées à la coordination, et non comme des membres d'un réseau.

Les coordinations départementales des musées ont désormais principalement un rôle de coordination culturelle du territoire. Elles sont de moins en moins responsables de sites et sont finalement assez peu portées sur la création d'échange. Elles mettent par contre en avant la mutualisation, le regroupement ; elles sont par ailleurs de plus en plus portées vers l'amélioration qualitative et la rationalisation de l'offre muséale. 

territoire d'aujourd'hui, les conservations départementales des musées sont clairement devenues aujourd'hui des coordinations départementales des musées.

\section{Méthodologie d'enquête}

La présente étude 9 sur les coordinations départementales des musées s'est déroulée sur un an et demi en trois étapes, dans le cadre du Master Expographie-Muséographie de l'université d'Artois. Une pré-enquête auprès de tous les départements métropolitains a d'abord été menée sous la forme d'une première prise de contact, puis une large enquête détaillée a été réalisée avec l'envoi aux quatre-vingt-seize départements d'un questionnaire informatique comportant quarante-sept questions. La quasi-totalité des départements a été interrogée, une soixantaine a répondu au questionnaire, dont une petite quarantaine de coordinations départementales des musées sur les quarante-sept recensées. Pour les autres, soit des entretiens téléphoniques ont eu lieu, soit la documentation interne et/ou externe du service a été étudiée.

49

Enfin, des entretiens spécifiques ont été conduits auprès de cinq coordinations départementales sélectionnées pour approfondir le sujet. Cette sélection ne présente pas des cas idéaux ou des cas critiques, mais des services représentatifs des fonctionnements des différentes coordinations départementales des musées. Ils ont été choisis après l'analyse du questionnaire informatique et grâce à ses conclusions. Les études de cas rédigées à la suite de ces entretiens ont été relues et approuvées par les responsables de chacune des coordinations. Une fois l'étude terminée, elle a été envoyée à toutes les coordinations départementales des musées.

\section{BIBLIOGRAPHIE}

Benneteu, B., Albertin, A.-M., Hue, M., Pelletier, A. et Rooryck, I. Les conservations départementales en Midi-Pyrénées, Musées et collections publiques de France, n² 267, 2013, pp. 17-21.

Denise, F., Joly, M.-H. et Deleplancque, R. Enquête sur les conservations départementales en France : état des lieux, missions - actions, politiques des publics, Paris, DMF, 1999, 112p.

Joly, M.-H. et Denise, F. Les conservations départementales, Musées et collections publiques de France , n' 226, 2000, pp. 32-38.

Leng, S. Synthèse de l'enquête sur les Conservations départementales en France, document interne au Conseil général du Jura, 2005, 27 p.

Musso, P. Télécommunications et philosophie des réseaux, La postérité paradoxale de Saint-Simon. Paris : PUF, 1997, 396 p.

Musso, P. Critique des réseaux, Paris, PUF, 2003, 375 p.

Pottier, J.-P. Les systèmes d'organisation administrative, les musées et les réseaux - Le cadre départemental : la Lozère, Musées et Collections publiques de France, n²26, 2000, pp. 39-42.

La Lettre de I'OCIM, 157 | 2015 
Roche-Bernard, G. Enquête sur les conservations départementales en France, document interne au Conseil général du Val-d'Oise, 1992, 24 p.

Sarret, F. Les conservations départementales des musées en Languedoc-Roussillon, Musées et Collections publiques de France, $\mathrm{n}^{\circ}$ 235-236, 2002, pp. 10-11.

Zacharie, E. Les musées en réseaux - Synthèse d'une étude de 1997, La Gazette des musées des Landes, $\mathrm{n}^{\circ}$ 5, octobre 2002, pp. 4-7.

Vital, C. Les conservations départementales : historique et évolution récente, Musées et collections publiques de France, $\mathrm{n}^{\circ}$ 267, 2013, pp. 17-21.

\section{NOTES}

1. Denise, F., Joly, M.-H. et Deleplancque, R. Enquête sur les conservations départementales en France : état des lieux, missions - actions, politiques des publics, Paris, DMF, 1999, $112 \mathrm{p}$.

2. Roche-Bernard, G. Enquête sur les conservations départementales en France, document interne au Conseil général du Val-d'Oise, 1992, 24 p.

3. Denise, F., op. cit.

4. Leng, S. Synthèse de l'enquête sur les Conservations départementales en France, document interne au Conseil général du Jura, 2005, 27 p.

5. La définition complète est "des collections, un territoire, un réseau, gérés par des équipes sur site, sous l'impulsion scientifique et technique d'un conservateur".

6. Pottier, J.-P. Les systèmes d'organisation administrative, les musées et les réseaux - Le cadre départemental : la Lozère, Musées et Collections publiques de France, n²26, 2000, pp. 39-42.

7. Musso, P. Télécommunications et philosophie des réseaux - La postérité paradoxale de Saint Simon, Paris, PUF, 1997, 396 p. et Critique des réseaux, Paris, PUF, 2003, 375 p.

8. Charie, E. Les musées en réseaux - Synthèse d'une étude de 1997, La Gazette des musées des Landes, $n^{\circ} 5$, octobre 2002 , pp. 4-7.

9. L'étude complète intitulée Les coordinations départementales des musées : des services au service du territoire - Enquête nationale est disponible en ligne sur www.calameo.com/ books/0024269358168c407c7eb, elle peut également être envoyée en contactant l'auteure. Elle contient, en plus de l'analyse de l'étude, des fiches détaillées sur chacune des coordinations départementales des musées présentant leur historique, leurs missions et leur fonctionnement.

\section{RÉSUMÉS}

Poursuivant le travail de deux enquêtes nationales menées par le ministère de la Culture en 1992 et 1999, l'auteure présente ici les résultats d'une recherche sur les conservations départementales des musées effectuée en 2013, analysant le contexte de leur création, leur fonctionnement et l'évolution de leurs missions ainsi que leur transformation progressive en organismes désormais chargés de la coordination culturelle du territoire. 
INDEX

Mots-clés : conservation, musée

\section{AUTEUR}

\section{CÉCILE MASSOT}

Muséographe

cecile.massot@gmail.com 